Article

\title{
Thrombotic Risk Detection in Patients with Polycythemia Vera: The Predictive Role of DNMT3A/TET2/ASXL1 Mutations
}

\author{
Adrián Segura-Díaz ${ }^{1}$, Ruth Stuckey ${ }^{1}{ }^{\circledR}$, Yanira Florido ${ }^{1}$, Jesús María González-Martín ${ }^{2}{ }^{\circledR}$, \\ Juan Francisco López-Rodríguez ${ }^{1}$, Santiago Sánchez-Sosa ${ }^{1}{ }^{\circledR}$, Elena González-Pérez ${ }^{1}$, \\ María Nieves Sáez Perdomo ${ }^{1}$, María del Mar Perera ${ }^{1}$, Silvia de la Iglesia ${ }^{1}$, \\ Teresa Molero-Labarta 1,3, María Teresa Gómez-Casares 1,3,* and Cristina Bilbao-Sieyro 1,4,* \\ 1 Hematology Department, Hospital Universitario de Gran Canaria Dr. Negrín, \\ 35019 Las Palmas de Gran Canaria, Spain; adsegura@hotmail.es (A.S.-D.); rstuckey@funcanis.es (R.S.); \\ floryyana@hotmail.com (Y.F.); juanfra5226@gmail.com (J.F.L.-R.); Jsanchez@funcanis.es (S.S.-S.); \\ gpelena88@gmail.com (E.G.-P.); marysnow@telefonica.net (M.N.S.P.); \\ mperalvm@gobiernodecanarias.org (M.d.M.P.); siglini@gobiernodecanarias.org (S.D.1.I.); \\ tmollab@gobiernodecanarias.org (T.M.-L.) \\ 2 Investigation Unit, Hospital Universitario de Gran Canaria Dr. Negrín, 35019 Las Palmas de Gran Canaria, \\ Spain; josu.estadistica@gmail.com \\ 3 Department of Medical Sciences, Universidad de Las Palmas de Gran Canaria, 35016 Las Palmas de Gran \\ Canaria, Spain \\ 4 Morphology Department, Universidad de Las Palmas de Gran Canaria, 35016 Las Palmas de Gran Canaria, \\ Spain \\ * Correspondence: mgomcasf@gobiernodecanarias.org (M.T.G.-C.); bilbaocristina@gmail.com (C.B.-S.); \\ Tel.: +34-928-479433 (M.T.G.-C. \& C.B.-S.)
}

Received: 17 February 2020; Accepted: 26 March 2020; Published: 10 April 2020

\begin{abstract}
The development of thrombotic events is common among patients with polycythemia vera (PV), essential thrombocythemia (ET), and primary myelofibrosis (PMF). We studied the influence of pathogenic mutations frequently associated with myeloid malignancies on thrombotic events using next-generation sequencing (NGS) in an initial cohort of 68 patients with myeloproliferative neoplasms (MPN). As expected, the presence of mutations in DNMT3A, TET2, and ASXL1 (DTA genes) was positively associated with age for the whole cohort ( $p=0.025$, OR: 1.047, 95\% CI: 1.006-1.090). Also, while not related with events in the whole cohort, DTA mutations were strongly associated with the development of vascular events in PV patients $(p=0.028)$. To confirm the possible association between the presence of DTA mutation and thrombotic events, we performed a case-control study on 55 age-matched patients with PV (including 12 PV patients from the initial cohort, 25 with event vs. 30 no event). In the age-matched case-control PV cohort, the presence of $\geq 1$ DTA mutation significantly increased the risk of a thrombotic event (OR: 6.333, $p=0.0024)$. Specifically, mutations in TET2 were associated with thrombotic events in the PV case-control cohort (OR: 3.56, 95\% CI: 1.15-11.83, $p=0.031$ ). Our results suggest that pathogenic DTA mutations, and particularly TET2 mutations, may be an independent risk factor for thrombosis in patients with PV. However, the predictive value of TET2 and DTA mutations in ET and PMF was inconclusive and should be determined in a larger cohort.
\end{abstract}

Keywords: prognosis; myeloproliferative neoplasms; cardiovascular disease; thrombosis; next-generation sequencing; personalized medicine 


\section{Introduction}

The BCR-ABL1-negative myeloproliferative neoplasms (MPN) polycythemia vera (PV), essential thrombocythemia (ET), and primary myelofibrosis (PMF) are rare hematological malignancies characterized by the clonal expansion of an abnormal hematopoietic stem cell. Patients with MPN show a highly variable prognosis. Adverse cardiovascular events including thrombosis and hemorrhages are common among patients with MPN, affecting 34-39\% of patients with PV, 10-29\% of patients with ET, and 7.2-13.2\% of patients with PMF [1]. Indeed, adverse cardiovascular events are the major cause of morbidity and mortality in patients with MPN.

Prognostic scores have been developed for each MPN subtype to stratify patients according to the risk of thrombosis and inform their treatment plan. For example, IPSET includes the JAK2V617F mutation, a confirmed thrombosis risk factor for ET patients [2]. Other mutations, such as CALR type I, have also been associated with an increased risk of thrombosis, while type II CALR mutations are associated with a lower incidence of thrombosis compared to JAK2 mutation [3]. In many cases, thrombotic events are the presenting feature of the disease at diagnosis. However, fatal and non-fatal thrombotic events are frequent during follow-up, even in patients treated with cytoreduction in combination with oral anticoagulants and/or anti-aggregant therapies [4]. Thus, existing algorithms to predict the development of thrombosis in MPN patients require improvements.

Advances in sequencing technology have improved our understanding of the contribution of non-driver mutations as risk factors for overall survival and fibrotic or leukemic transformation. Moreover, the inclusion of somatic mutations in the risk classification of MPN has contributed to the development of more accurate predictive tools [5], such as the mutation-enhanced MIPSS70+ prognostic score for PMF [6] and the online calculator of individualized MPN patient outcomes [7].

Sequencing studies have also demonstrated a direct association between age and the accumulation of somatic mutations, particularly in DNMT3A, TET2, and ASXL1, often collectively referred to as the DTA genes, as well as JAK2 [7-12]. Indeed, DTA gene mutations have been shown to confer a selective advantage and lead to clonal hematopoiesis (CHIP) [8-11]. Importantly, individuals with clonal hematopoiesis (even those without a hematologic malignancy) are estimated to have a 1.9-fold higher risk of developing coronary disease and a 4-fold higher risk of myocardial infarction [12].

Despite the recent advances in sequencing techniques and an increased interest in the contribution of non-driver mutations as risk factors, to date, very few studies have investigated the association between mutations in non-driver genes and the development of thrombosis in MPN patients. To address this research need, we aimed to study the impact of gene mutations frequently associated with myeloid malignancies on thrombotic events using next-generation sequencing in a cohort of $B C R$ - $A B L 1$-negative MPN patients.

\section{Results}

The initial cohort included a total of 68 patients, consisting of 16 patients with PV, 25 with ET, 16 with PMF, and 11 patients with secondary myelofibrosis (SMF). Patients had an average age of 68 years (range 43-90; median 68 years) and $38.2 \%$ were males (patient characteristics are shown in Supplementary Table 1). With a median follow-up of 50 months (range 1-200 months), 22 patients developed 25 thrombotic events after diagnosis (32.4\% of the MPN cohort), 12 corresponding to arterial thrombosis (48.0\% of the total thrombotic episodes) and 13 to venous thrombosis (Table 1 ). Events were registered in $43.8 \%$ of patients with PV (7/16 patients), $40 \%$ of patients with ET (10/25 patients), and $18.5 \%$ (5/27 patients) with MF. 
Table 1. Arterial and venous thrombotic events registered in the myeloproliferative neoplasms (MPN) cohort at or after diagnosis.

\begin{tabular}{|c|c|c|c|}
\hline & MPN & First Event & Second Event \\
\hline 1 & PV & IS & \\
\hline 2 & PV & RVO & \\
\hline 3 & PV & MI & \\
\hline 4 & PV & Budd-Chiari & \\
\hline 5 & PV & MI & \\
\hline 6 & PV & DVT & TIA \\
\hline 7 & PV & DVT and PE & \\
\hline 8 & ET & PVT & \\
\hline 9 & ET & MI & \\
\hline 10 & ET & $\mathrm{PE}$ & \\
\hline 11 & ET & Budd-Chiari & \\
\hline 12 & ET & Acute carotid stroke & \\
\hline 13 & ET & TIA & \\
\hline 14 & ET & DVT & \\
\hline 15 & ET & IS & MI \\
\hline 16 & ET & MI & \\
\hline 17 & ET & SVT & \\
\hline 18 & PMF & PVT & \\
\hline 19 & PMF & PVT & \\
\hline 20 & PMF & MI & \\
\hline 21 & PMF & PE & \\
\hline 22 & SMF (post-PV) & PE & PVT \\
\hline
\end{tabular}

IS: ischemic stroke, RVO: retinal vein occlusion, MI: myocardial infarction, DVT: deep vein thrombosis, PE: pulmonary embolism, TIA: transient ischemic attack, PVT: portal vein thrombosis, SVT: superficial vein thrombosis.

Among the patients with MPN, 48.5\% (33/68 patients) presented a pathogenic non-driver mutation (i.e., in genes other than JAK2, CALR, or MPL; Table S2). The mean number of pathogenic non-driver mutations was 0.63 mutations/patient; $11 \%$ of patients presented with more than one pathogenic non-driver mutation. The most frequently mutated genes were TET2 $(32.6 \%), \operatorname{ASXL1}(14.0 \%)$, and DNMT3A (14.0\%) (Figure 1A).The presence of mutations in the CHIP-associated DTA genes was $62.5 \%$ in PV, 56.3\% in PMF, $45.5 \%$ in SMF, and $12 \%$ in ET (Figure 1B-E).
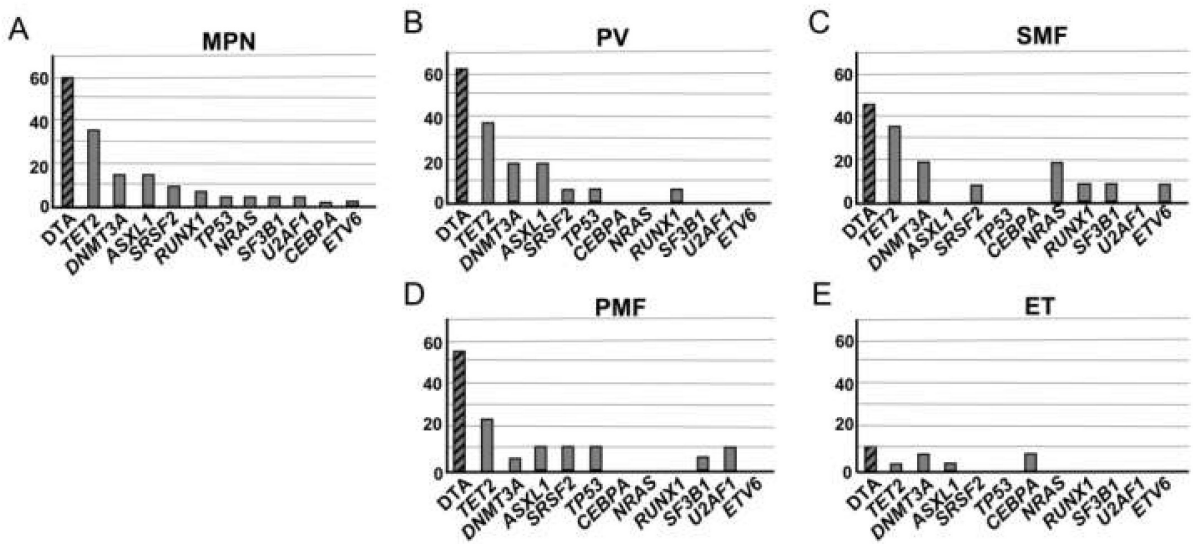

Figure 1. Most frequent non-driver gene mutations. (A) For the whole patient cohort $(n=68)$, and for each neoplasia separately, (B) polycythemia vera (PV) $(n=16),(\mathbf{C})$ secondary myelofibrosis (SMF) $(n=11),(\mathrm{D})$ primary myelofibrosis (PMF) $(n=16)$, and (E) essential thrombocythemia (ET) $(n=25)$. DTA genes (DNMT3A, TET2, ASXL1) were the most frequently mutated in both the whole cohort and individual neoplasias. Hatched bars represent the sum of mutations in the DTA genes. 
As expected, we observed a positive association between the presence of DTA mutations and age ( $p=0.025$, OR: $1.047,95 \%$ CI: 1.006-1.090). In the whole series, the presence of DTA mutations or any non-driver mutation were not related with the development of thrombotic events. However, when testing each neoplasia separately, a significant association between the variables of presence of a DTA mutation and vascular event was observed in PV patients $\left(p=0.031\right.$, Pearson's $\left.\chi^{2}\right)$. Specifically, $85.7 \%$ of patients with PV and an event (6/7 patients) harbored a DTA mutation compared to $33.3 \%$ of patients with PV without an event (3/9 patients). When also considering SMF-post PV (6 additional patients, 1 of whom had an event), $87.5 \%$ (7/8 patients) with an event had a DTA mutation compared to $42.9 \%$ (6/14 patients) without an event ( $p=0.028$, Fisher's exact test).

The association observed between presence of DTA mutation and thrombotic event in PV patients but not in the whole MPN series could be conditioned by the co-occurrence of a JAK2 mutation (since almost all patients with PV have $J A K 2$ mutations compared to 50\% in patients with MF, 60\% in patients with SMF post-ET, and $48 \%$ in patients with ET in our series). To analyze this possibility, we first evaluated if there was an association between the presence of a JAK2 mutation and thrombotic event in patients with ET, PMF, and SMF post-ET $(n=44$ patients with information available about thrombotic events). Using Pearson's $\chi^{2}$ test we observed an association between the presence of a JAK2 mutation and thrombotic event in these patients, albeit not statistically significant ( $p=0.124$, Table S3).We were unable to determine if an association existed between a thrombotic event and the presence of a DTA mutation or any non-driver mutation comparing JAK2-mutated versus JAK2-wild-type patients due to the low number of events (Table S4).

To confirm the possible association between the presence of DTA mutation and thrombotic events in PV, we performed a case-control study on 55 age-matched patients (Figure S1) with PV (including 12 of the 16 PV patients from the initial cohort). Of the 55 age-matched patients, 25 experienced a thrombotic event (including 15 events pre-diagnosis or at diagnosis and 16 events post-diagnosis) vs. $30 \mathrm{PV}$ patients without an event (Table S5). The median follow-up of patients with an event was 108.3 months (P25-P75 44.3-163.0) and 120.6 months (P25-P75 56.2-164.8) for patients without an event.

Following targeted next-generation sequencing (NGS) with the myeloid gene panel, we observed a significant association between the variables of thrombotic event and presence of a DTA mutation for the PV age-matched cohort ( $p=0.0024$, OR: 6.33, 95\% CI: 2.02-22.32, Fisher's exact test; Figure 2): 65.5\% of PV patients with an event harbored a DTA mutation vs. 34.5\% of PV patients without an event.

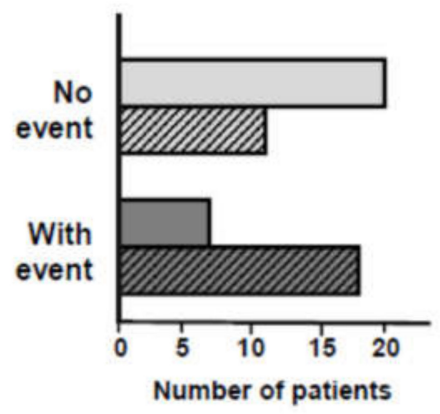

\begin{tabular}{|c|c|c|c|c|}
\hline Group & $n$ & $\begin{array}{l}\text { No DTA } \\
\text { mutation (\%) }\end{array}$ & $\begin{array}{l}\text { DTA } \\
\text { mutated }(\%)\end{array}$ & $p$ value \\
\hline No event & 30 & $20(76.9)$ & $10(34.5)$ & \multirow[t]{2}{*}{0.0024} \\
\hline With event & 25 & $6(23.1)$ & $19(65.5)$ & \\
\hline Total & 55 & 26 & 29 & \\
\hline
\end{tabular}

Figure 2. Frequency of DTA mutations in the no thrombotic event and with thrombotic event groups of PV patients. A significant association was observed between event and the presence of DTA mutation. Open bars represent no DTA mutation, hatched bars represent a DTA mutation. DTA: DNMT3A, TET2, ASXL1. Odds ratio 4.68 (95\% confidence interval 1.493-14.644).

Considering mutations in the TET2 gene in isolation, a positive association existed between thrombotic event and the presence of a pathogenic TET2 mutation ( $p=0.031$, OR: 3.56, 95\% CI: 1.15-11.83, Fisher's exact test; Figure 3): $65.0 \%$ of PV patients with an event harbored a TET2 mutation vs. $35.0 \%$ of PV patients without an event. A significant association was also observed between the presence of any pathogenic non-driver mutation and thrombotic event ( $p=0.0077, \mathrm{OR}: 5.23,95 \% \mathrm{CI}$ : 
1.63-19.23, Fisher's exact test; Figure S2). However, when patients with TET2 mutations were removed and the logistic regression analysis repeated, the association of thrombotic event with ASXL1/DNMT3A mutations or any non-driver mutation (other than TET2) lost statistical significance $(p=0.185$ and $p=0.487$, respectively).

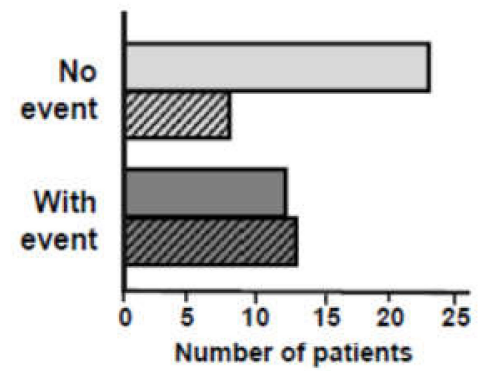

\begin{tabular}{|l|l|l|l|l|}
\hline Group & $n$ & $\begin{array}{l}\text { No TET2 } \\
\text { mutation (\%) }\end{array}$ & $\begin{array}{l}\text { TET2 } \\
\text { mutated (\%) }\end{array}$ & \multirow{2}{*}{0 value } \\
\hline No event & 30 & $23(65.7)$ & $7(35.0)$ & 0.031 \\
\hline With event & 25 & $12(34.3)$ & $13(65.0)$ & \\
\hline Total & 55 & 35 & 20 & \\
\hline
\end{tabular}

Figure 3. Frequency of TET2 mutations in the no thrombotic event and with thrombotic event groups of PV patients. Open bars represent no TET2 mutation, hatched bars represent TET2 mutation. Odds ratio 3.11 (95\% confidence interval 1.012-9.582).

\section{Discussion}

Our study used next-generation sequencing to investigate the impact of pathogenic non-driver mutations commonly associated with myeloid malignancies on thrombotic risk. These mutations were harbored by $48.5 \%$ of the MPN patient cohort, in raccordance with the previously observed prevalence of 53\% for patients with PV or ET [2]. The CHIP-associated DTA genes were the most frequently mutated in the whole MPN cohort, also in accordance with previously published results $[7,13]$. The frequency of CHIP-associated DTA gene mutations ranged from $12 \%$ for patients with ET to $62.5 \%$ for patients with PV. In contrast, the estimated frequency is $2-3 \%$ in the peripheral blood of healthy individuals, rising to approximately $10 \%$ in those aged over 70 years [9].

In patients with ET, PMF, and SMF post-ET, the presence of JAK2 mutation was marginally associated with thrombotic event. However, since practically all PV patients were mutated in JAK2, it is clear that an additional factor must contribute to thrombotic risk in patients with PV. Indeed, our preliminary results reveal that mutations in DNMT3A, TET2, and ASXL1 genes, while not related with thrombotic events in the overall cohort, were strongly associated with events in PV patients. The presence of at least one DTA mutation increased the risk of developing a vascular event by 6-fold in patients with PV. Specifically, mutations in the TET2 gene in isolation were also associated with thrombosis in the PV case-control cohort. A previous study reported a possible association between TET2 mutations and thrombosis in ET [13]. However, a 2017 study which investigated the impact of mutations in JAK2, TET2, and ASXL1 genes on thrombotic risk in 587 patients with PV [14] found that TET2 or ASXL1 mutations (detected in $18 \%$ and $11 \%$ of patients, respectively) did not impact arterial nor venous thrombosis [14], although this was not a case-control study.

Our results are in accordance with recent evidence suggesting that the increased cardiovascular risk associated with mutations in genes encoding the epigenetic regulators DNMT3A, TET2, and ASXL1 is due to altered methylation, which causes the increased transcription of pro-inflammatory genes and may trigger atherosclerosis [12,15,16]. For example, a 2012 study showed that loss of TET2 function was associated with atherosclerosis and the increased secretion of pro-inflammatory factors including IL-1 $\beta$ [15]. In our study, TET2 mutation seems to be a key factor in driving thrombotic events in PV. A larger series would be needed to determine the contribution of DNMT3A, ASXL1, or other non-driver genes to the risk of developing an adverse cardiovascular event in patients with PV. Moreover, the predictive value of TET2 and DTA mutations on thrombotic risk in patients with ET and PMF was inconclusive due to the low number of events and the small number of analyzed patients. This association should be determined in larger ET and PMF cohorts and further case-control studies. 
Our observations, if confirmed in a larger patient series, could help to identify PV patients at diagnosis who are at higher risk of developing thrombosis. For example, the current risk algorithm for patients with PV, stratified into low risk ( $<60$ years, no history of thrombosis) and high risk ( $>60$ years and/or history of thrombosis) could be modified to include a third very high-risk group ( $>60$ years and/or history of thrombosis with a TET2 mutation). Such very high-risk patients would benefit from closer monitoring and a modified treatment plan, with the aim of reducing the currently high rates of thrombotic events among MPN patients. For example, preventative therapies with anti-inflammatory agents such as anti-IL-1 $\beta$ might be effective [16]. Randomized clinical trials are required to confirm whether such agents would prevent adverse cardiovascular events in high-risk patients with MPN, although a preliminary study involving patients with CHIP enrolled in the CANTOS study reported that patients with TET2 mutations responded better to the anti-IL-1 $\beta$ antibody canakinumab than patients without CHIP-associated mutations [17]. It would also be interesting to evaluate if treatment with ruxolitinib, an agent with anti-inflammatory and immunosuppressive properties, can prevent thrombosis in patients with MPN given the reduction of pro-inflammatory cytokines, including IL-6, as a result of its JAK1 inhibitory action [18].

DNA sequencing of patients with MPN using a relatively-inexpensive standardized myeloid panel is not currently part of routine clinical practice. However, such an analysis can provide important prognostic information on vascular risk and overall survival [19].

To conclude, given the continued aging of the global population (with adults aged 65 and over estimated to constitute $16 \%$ of the global population by 2050 [20]), the high incidence of MPNs in the over-65 age group, the increasing incidence of DTA mutations with age [7-11] and the association of DTA mutations with both MPN and cardiovascular risk [12], the observation that DTA mutations (especially mutations in TET2) may be an independent risk factor for thrombosis in PV is particularly pertinent. It also confirms the importance of a multidisciplinary cardio-onco-hematology approach for the management of patients with MPN.

\section{Materials and Methods}

\subsection{Ethical Approval}

This retrospective non-interventional study was conducted in accordance with the Declaration of Helsinki, and the protocol was approved by our institutional review board (Comité Ético de Investigación Clínica, approval no. ref. 2019-230-1). Informed consent for inclusion was provided by all patients before they participated in the study.

\subsection{Patients}

Patients aged 18 years and above with confirmed diagnosis of PV, ET, or MF according to the 2016-revised WHO criteria were eligible for inclusion. Informed consent was obtained for a total of 68 patients with MPN (initial MPN series)and 55 age-matched PV patients (PV case-control cohort, including 12 of the 16 PV patients from the initial MPN cohort) diagnosed between January 2003 and July 2018 at the Hospital Universitario de Gran Canaria Dr. Negrín, Las Palmas, Spain.

The MPN series included 16 PV, 25 ET, 16 PMF, and 11 SMF patients. Patients with MF were taken consecutively; the PV and ET patients were retrospectively chosen from a previous research project (with different objectives to this study) and the status of the patients' thrombotic events was unknown at the time of their selection.

For the case-control cohort, all patients diagnosed at our hospital with a confirmed diagnosis of $\mathrm{PV}$ and thrombotic events in their patient history were taken as "cases" $(n=25)$. Control patients (with a diagnosis of PV but no thrombotic event) were taken consecutively and age-matched with the cases $(n=30)$. Control patients that did not age-match with a control were removed.

See Tables S1 and S5 for patient characteristics of the initial MPN cohort and PV case-control cohort, respectively. 


\subsection{Next-Generation Sequencing (NGS)}

NGS was performed on $200 \mathrm{ng}$ genomic DNA extracted from peripheral blood at diagnosis. Sequencing was performed using a MiSeq (Illumina, San Diego, CA, USA) platform with the targeted 30-gene panel Myeloid Tumor Solution ${ }^{\mathrm{TM}}$ (SOPHiA Genetics, Saint-Sulpice, Switzerland). Variants were identified using the analysis software SOPHiA DDM (4.8.1.3). Only variants with an allelic frequency (VAF) $\geq 2 \%$ (established as cutoff for mutations associated with CHIP in peripheral blood [6]), a described population frequency $(\mathrm{MAF})<1 \%$, and an annotated pathogenic effect (or probability $>90 \%$ of being pathogenic) were included, with pathogenicity determined according to ClinVar (www.ncbi.nlm.nih.gov/clinvar/), COSMIC (https://cancer.sanger.ac.uk/cosmic), VarSome (https:// varsome.com/), ENSEMBL (https://www.ensembl.org/index.html), and IARC TP53 databases (http: //p53.iarc.fr/TP53GeneVariations.aspx), and published studies.

\subsection{Statistical Analysis}

Chi-squared tests and logistic regression analyses were performed to analyze the impact of the independent variables on thrombotic risk. Odd-ratios (OR) with correspondent $p$ values and confidence intervals (CI) were estimated. All $p$ values $<0.05$ were considered statistically significant. Analyses were performed using statistical software R Core Team 2020, version 3.6.2.

\section{Conclusions}

Our study investigated the association between pathogenic mutations in non-driver genes and the development of thrombosis, the major cause of morbidity and mortality in patients with $B C R-A B L 1$-negative MPN. Pathogenic DTA mutations increased thrombotic risk by 6-fold in PV patients, although the association with patients with MF or ET was inconclusive. Mutations in TET2 seemed to be a key factor in driving thrombotic events in PV. Our results are in accordance with recent evidence linking TET2 mutations with atherosclerosis and increased cardiovascular risk. Our observations could be applied to improve current risk algorithms for the more accurate identification of PV patients at diagnosis who are at higher risk of developing thrombosis.

Supplementary Materials: The following are available online at http://www.mdpi.com/2072-6694/12/4/934/s1. Figure S1: Box and whisker plot of age-matched PV patient case-control study cohort, Figure S2: Frequency of non-driver gene mutations in the no thrombotic event and with thrombotic event groups of PV patients, Table S1: Patient characteristics of whole MPN n $=68$., Table S2: Frequency of mutation in 1 of the 30 genes included in the myeloid panel (including the driver genes $J A K 2, C A L R$, and MPL) for the whole MPN patient series, Table S3: Contingency table for Pearson's $\chi 2$ test to evaluate the association between JAK2 mutations and thrombotic events in patients with ET, PMF, and SMF post-ET $(n=44)$, Table S4: Contingency tables for Pearson's $\chi 2$ test to evaluate the association between thrombotic events and DTA mutations or any non-driver mutations in JAK2-mutated versus JAK2 wild-type patients with ET, PMF, and SMF post-ET $(n=44)$, Table S5: Patient characteristics of the age-matched PV case-control cohort.

Author Contributions: Conceptualization, A.S.-D., M.T.G.-C., and C.B.-S.; Data curation, A.S.-D., R.S., S.S.-S., E.G.-P., and M.N.S.P.; Formal analysis, J.M.G.-M. and C.B.-S.; Funding acquisition, R.S. and C.B.-S.; Investigation, A.S.-D., R.S., J.F.L.-R., M.d.M.P., S.d.1.I., T.M.-L., M.T.G.-C., and C.B.-S.; Methodology, Y.F. and C.B.-S.; Project administration, R.S., M.T.G.-C., and C.B.-S.; Resources, T.M.-L. and M.T.G.-C.; Supervision, M.T.G.-C. and C.B.-S.; Writing—original draft, R.S., M.T.G.-C., and C.B.-S.; Writing-review and editing, A.S.-D., R.S., Y.F., J.M.G.-M., J.F.L.-R., S.S.-S., E.G.-P., M.N.S.P., M.d.M.P., S.d.l.I., T.M.-L., M.T.G.-C., and C.B.-S. All authors have read and agreed to the published version of the manuscript.

Funding: This work received funding from the Colegio de Médicos de Las Palmas (Beca I+E 2018), Fundación Canaria de Investigaciones Sanitarias (PIFUN62/17), and Fundación DISA (OA 15/005, OA/19).

Conflicts of Interest: The authors have no competing interests to declare. 


\section{References}

1. Tefferi, A.; Elliott, M. Thrombosis in myeloproliferative disorders: Prevalence, prognostic factors, and the role of leukocytes and JAK2V617F. Semin. Thromb. Hemost. 2007, 33, 313-320. [CrossRef] [PubMed]

2. Barbui, T.; Finazzi, G.; Carobbio, A.; Thiele, J.; Passamonti, F.; Rumi, E.; Ruggeri, M.; Rodeghiero, F.; Randi, M.L.; Bertozzi, I.; et al. Development and validation of an International Prognostic Score of thrombosis in World Health Organization-essential thrombocythemia (IPSET-thrombosis). Blood 2012, 120, 5128-5133. [CrossRef] [PubMed]

3. Pietra, D.; Rumi, E.; Ferretti, V.V.; Di Buduo, C.A.; Milanesi, C.; Cavalloni, C.; Sant'Antonio, E.; Abbonante, V.; Moccia, F.; Casetti, I.C.; et al. Differential clinical effects of different mutation subtypes in CALR-mutant myeloproliferative neoplasms. Leukemia 2016, 30, 431-438. [CrossRef] [PubMed]

4. De Stefano, V.; Za, T.; Rossi, E.; Vannucchi, A.M.; Ruggeri, M.; Elli, E.; Micò, C.; Tieghi, A.; Cacciola, R.R.; Santoro, C.; et al. Recurrent thrombosis in patients with polycythemia vera and essential thrombocythemia: Incidence, risk factors, and effect of treatments. Haematologica 2008, 93, 372-380. [CrossRef] [PubMed]

5. Tefferi, A.; Vannucchi, A.M. Genetic risk assessment in myeloproliferative neoplasms. Mayo Clin. Proc. 2017, 92, 1283-1290. [CrossRef] [PubMed]

6. Tefferi, A.; Guglielmelli, P.; Lasho, T.L.; Gangat, N.; Ketterling, R.P.; Pardanani, A.; Vannucchi, A.M. MIPSS70+ Version 2.0: Mutation and karyotype-enhanced international prognostic scoring system for primary myelofibrosis. J. Clin. Oncol. 2018, 36, 1769-1770. [CrossRef] [PubMed]

7. Grinfeld, J.; Nangalia, J.; Baxter, E.J.; Wedge, D.C.; Angelopoulos, N.; Cantrill, R.; Godfrey, A.L.; Papaemmanuil, E.; Gundem, G.; MacLean, C.; et al. Classification and personalized prognosis in myeloproliferative neoplasms. N. Engl. J. Med. 2018, 379, 1416-1430. [CrossRef] [PubMed]

8. Laurie, C.C.; Laurie, C.A.; Rice, K.; Doheny, K.F.; Zelnick, L.R.; McHugh, C.P.; Ling, H.; Hetrick, K.N.; Pugh, E.W.; Amos, C.; et al. Detectable clonal mosaicism from birth to old age and its relationship to cancer. Nat. Genet. 2012, 44, 642-650. [CrossRef] [PubMed]

9. Jaiswal, S.; Fontanillas, P.; Flannick, J.; Manning, A.; Grauman, P.V.; Mar, B.G.; Lindsley, R.C.; Mermel, C.H.; Burtt, N.; Chavez, A.; et al. Age-related clonal hematopoiesis associated with adverse outcomes. N. Engl. J. Med. 2014, 371, 2488-2498. [CrossRef] [PubMed]

10. Genovese, G.; Kähler, A.K.; Handsaker, R.E.; Lindberg, J.; Rose, S.A.; Bakhoum, S.F.; Chambert, K.; Mick, E.; Neale, B.M.; Fromer, M.; et al. Clonal hematopoiesis and blood-cancer risk inferred from blood DNA sequence. N. Engl. J. Med. 2014, 371, 2477-2487. [CrossRef] [PubMed]

11. Steensma, D.P.; Bejar, R.; Jaiswal, S.; Lindsley, R.C.; Sekeres, M.A.; Hasserjian, R.P.; Ebert, B.L. Clonal hematopoiesis of indeterminate potential and its distinction from myelodysplastic syndromes. Blood 2015, 126, 9-16. [CrossRef] [PubMed]

12. Jaiswal, S.; Natarajan, P.; Silver, A.J.; Gibson, C.J.; Bick, A.G.; Shvartz, E.; McConkey, M.; Gupta, N.; Gabriel, S.; Ardissino, D.; et al. Clonal hematopoiesis and risk of atherosclerotic cardiovascular disease. N. Engl. J. Med. 2017, 377, 111-121. [CrossRef] [PubMed]

13. Tefferi, A.; Lasho, T.L.; Guglielmelli, P.; Finke, C.M.; Rotunno, G.; Elala, Y.; Pacilli, A.; Hanson, C.A.; Pancrazzi, A.; Ketterling, R.P.; et al. Targeted deep sequencing in polycythemia vera and essential thrombocythemia. Blood Adv. 2016, 1, 21-30. [CrossRef] [PubMed]

14. Cerquozzi, S.; Barraco, D.; Lasho, T.; Finke, C.; Hanson, C.A.; Ketterling, R.P.; Pardanani, A.; Gangat, N.; Tefferi, A. Risk factors for arterial versus venous thrombosis in polycythemia vera: A single center experience in 587 patients. Blood Cancer J. 2017, 7, 662. [CrossRef] [PubMed]

15. Fuster, J.J.; MacLauchlan, S. Clonal hematopoiesis associated with TET2 deficiency accelerates atherosclerosis development in mice. Science 2017, 355, 842-847. [CrossRef] [PubMed]

16. Ridker, P.M.; MacFadyen, J.G.; Thuren, T.; Libby, P. Residual inflammatory risk associated with interleukin-18 and interleukin- 6 after successful interleukin-1b inhibition with canakinumab: Further rationale for the development of targeted anti-cytokine therapies for the treatment of atherothrombosis. Eur. Heart J. 2019, ehz542. [CrossRef] [PubMed]

17. Svensson, E.C.; Madar, A.; Campbell, C.D.; He, Y.; Sultan, M.; Healey, M.L.; D’Aco, K.; Fernandez, A.; Wache-Mainier, C.; Ridker, P.M.; et al. Abstract 15111: TET2-Driven Clonal Hematopoiesis Predicts Enhanced Response to Canakinumab in the CANTOS Trial: An Exploratory Analysis. Circulation 2018, 138, A15111. 
18. Elli, E.M.; Baratè, C.; Mendicino, F.; Palandri, F.; Palumbo, G.A. Mechanisms Underlying the Anti-inflammatory and Immunosuppressive Activity of Ruxolitinib. Front. Oncol. 2019, 9, 1186. [CrossRef] [PubMed]

19. Palumbo, G.A.; Stella, S.; Pennisis, M.S.; Pirosa, C.; Fermo, E.; Fabris, S.; Cattaneo, D.; Iurlo, A. The Role of New Technologies in Myeloproliferative Neoplasms. Front. Oncol. 2019, 9, 321. [CrossRef] [PubMed]

20. United Nations, Department of Economic and Social Affairs, Population Division. World Population Prospects 2019: Data Booklet. ST/ESA/SER.A/424. 2019. Available online: https://population.un.org/wpp/ (accessed on 2 October 2019).

C 2020 by the authors. Licensee MDPI, Basel, Switzerland. This article is an open access article distributed under the terms and conditions of the Creative Commons Attribution (CC BY) license (http://creativecommons.org/licenses/by/4.0/). 